\title{
Using a Complex Network Methodology to Track, Evaluate, and Transform Fundamental Care
}

\author{
Maria A PINERO DE PLAZA ${ }^{\mathrm{a}, \mathrm{b}, 1}$, Tiffany CONROY ${ }^{\mathrm{a}}$, \\ Alexandra MUDD ${ }^{\mathrm{a}}$ and Alison KITSON ${ }^{\mathrm{a}, \mathrm{b}}$ \\ ${ }^{a}$ Caring Futures Institute (CFI), College of Nursing and Health Sciences \\ Flinders University, Bedford Park, South Australia 5042 \\ ${ }^{\mathrm{b}}$ National Health and Medical Research Council, Transdisciplinary Centre of \\ Research Excellence in Frailty and Healthy Ageing, Adelaide, South Australia
}

\begin{abstract}
In this study, we drew on methods originating in complex adaptive systems and social network analysis to develop a novel way to quantify fundamental care. Data were obtained from a public statement from the Australian Royal Commission into Aged Care Quality and Safety. Results support the importance of using a systemic approach to assess the multiple dimensions of the fundamentals of care. Our method allows measurement of the problem within its system, providing a detailed quantification of care events and identifying excellence and improvement opportunities. We illustrate the strengths of this approach using principal component analysis and heat mapping. The application of the proposed methodology in healthcare decision-making, planning, and quality improvement is discussed.
\end{abstract}

Keywords. Complex adaptive systems, network, care, Fundamentals of Care Framework

\section{Introduction}

The healthcare system faces increasingly complex challenges that are shifting the nature in which health, caring, and nursing practices are measured [1]. These circumstances are opening the discussion about new ways to recognise, map and improve the applied science of nursing and the conceptual and contextual mechanisms behind such complex adaptive systems and networks [2,3]. Many new and useful complex methods and theories relevant for facing current health challenges are rooted in mathematics, engineering, and computer science, and therefore use specialised language and complicated formulas that historically are not friendly to other disciplines [4]. These characteristics can make complex networks approaches uninviting [4], but in this paper, we take away such scientific jargon and demystify complexity methods by introducing a novel approach to explore a complex healthcare problem and envision a methodological technique that could lend itself to current and future technological innovations and products.

\footnotetext{
${ }^{1}$ Corresponding Author, Maria A Pinero de Plaza, Caring Futures Institute (CFI), College of Nursing and Health Sciences, Flinders University, Bedford Park, South Australia 5042; e-mail: alejandra.pinerodeplaza@flinders.edu.au.
} 


\section{Aged Care Quality and Safety as a Complex Research Problem}

When care goes wrong costly assessments are undertaken to provide valuable information about the main issues behind the problem. Powerful illustrations of care going wrong are publicly available in Australia since the establishment of a Royal Commission into Aged Care Quality and Safety, in 2018. The Royal Commission interim report: "Neglect", [5] describes the lack of measurable care practices that comprise employing sufficient trained staff and creating a professional and caring culture that supports positive relationships between staff-consumer, staff-relative and staff-staff $[6,7]$.

Tracking and measuring care inner factors and networks is necessary because the real-life experience produces divergent agendas for different individuals and groups within the care system, [8] and rarely current measurements uncover and track such interrelations [9]. Gauging those interconnected issues can significantly transform the care system and its outcomes [9]. Therefore, we deliver our complex network examination of one Royal Commission' statement to demonstrate a way to track main issues and their interactions and prominence. The theoretical and evidence-based foundation of our approach is 'The Fundamentals of Care Framework' (FoC). We operationalised this validated framework because it focuses on a person's essential needs and relationship with staff to ensure their physical and psychosocial wellbeing, considering the system' external context and structures [10,7], as per Figure 1.

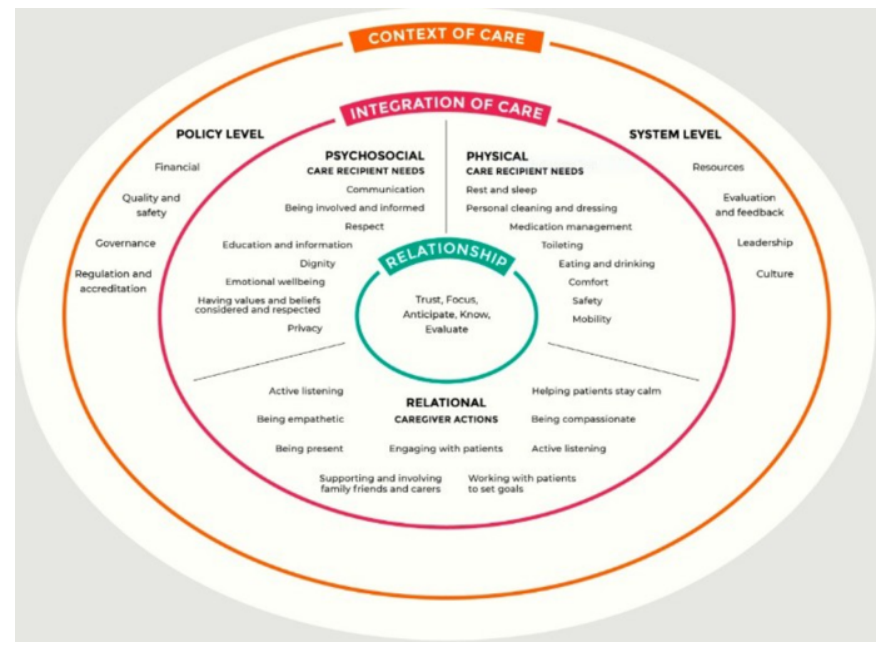

Figure 1. The Fundamentals of Care Framework [7] and FoC's definitions.

The FoC has three core dimensions: 1. Relationship (based on trust, and skills as anticipate, focus, know and evaluate); 2. Integration of care (psychosocial, physical, and relational needs); 3. Context of care (incorporating system and policy level factors) [7]. This complex system of three dimensions was co-developed and refined by an international group of clinicians, researchers, health service managers, policymakers, and consumers. FoC opens a view of the inter-dimensionalities between 38 subcomponents of care within its 3 dimensions. When care is delivered considering this holistic manner a high-quality service can be optimally provided $[7,10]$. 


\section{Methodology}

The proposed method is scientifically underpinned by graph theory [11], which is a branch of mathematics that is concerned with networks of points (e.g., points representing events or issues) linked by lines (representing their connexions). When any two points are joined one or more times, the graph can be represented as a network showing the 'weight' or number of connections between points (as per Figure 2 left image). The same data can be displayed in an adjacency matrix (as per Figure 2 right image). Such a matrix can be used to collect and analyse extensive network data.
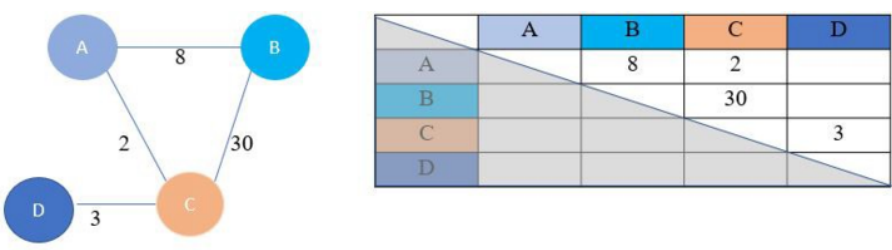

Figure 2. Network and adjacency table (weighted).

The conversion of the network to a matrix involves assigning, to each point of the network, a column and a row, and each combination of column and row is called a cell. A cell that contains empty spaces in our matrix is reflecting no connexions between row point and column point. Normally, where there is a connexion, the corresponding cell is given a number that indicates a frequency of connexion. We use the upper right triangle of the matrix to quantify our data to omit repetition on the lower side. We applied the FoC to the abovementioned graph theory, outlining its three dimensions and assigning each of the 38 elements as a point of interest, therefore creating a matrix with 38 rows by 38 columns [12]

\subsection{Data}

We undertook our complexity analysis using data from the statement of Ms Merle Mitchell, a resident in an aged care facility, who provided open and public direct evidence to the Royal Commission on the 6th May 2019 [12] (https://agedcare.royalcommission.gov.au/system/files/2020-06/WIT.0107.0001.0001.pdf).

\subsection{Procedure}

We (researchers from different disciplines) read the statement collectively, paragraph by paragraph, and looked for connexions of row point and column point, across the matrix. This involved imputing data following 6 key questions to act as prompts per each paragraph (comparing the 3 main dimensions of the $\mathrm{FoC}$ to each other), e.g. Is there any connexion between issues of the context of care and context of care? And then look for issues of the context of care and integration of care, and so on. This required noting two types of connexions: positive -good narratives of $\mathrm{FoC}$ - and negative -poor narratives of FoC- (as explained in this publication [12]). This matrix was also imputed in UCINET software for network analysis and depiction. 


\section{Results and Conclusion}

The statement of Ms Merle Mitchell analysed via The FoC Matrix shows the significance of interpersonal relationships across all dimensions of fundamental care [12]. The matrix highlights the value of contextual factors like 'resources and 'culture' having a severe effect on all aspects of care particularly the integration of care ('listening' and eating and drinking') affecting 'comfort', 'emotional wellbeing' and 'relationship' aspects ('trust', 'evaluate', 'know'). The context dimension was affecting the 'communication' and with that impacting on 'dignity', 'respect', 'being informed and involved' and other relevant connexions which are quantified and structurally reflected via a principal components' analysis, as per Figure 3.

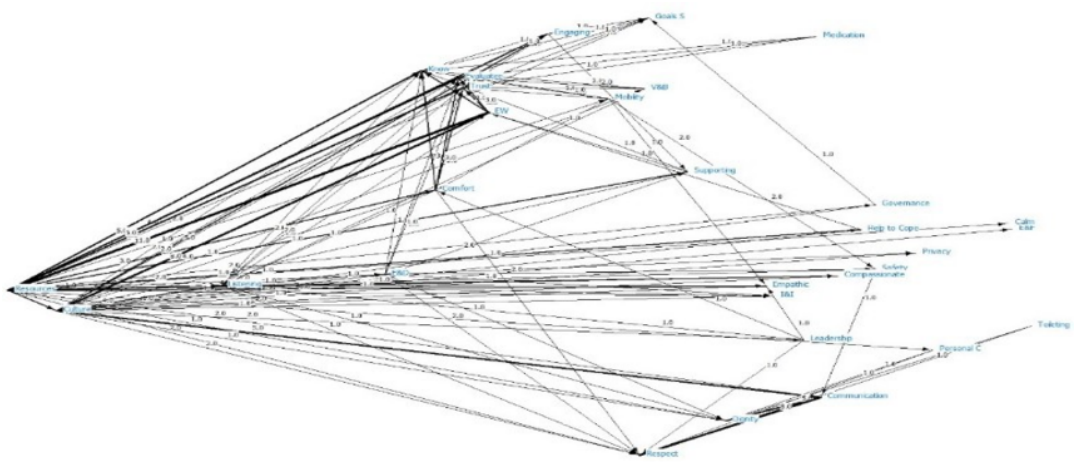

Figure 3. Ms Merle Mitchell statement analysed via a principal components' analysis.

Figure 3 demonstrates that our method moves beyond counting regular frequencies of issues, it is identifying interaction and prominence of connexions of FoC subdimensions and their granular elements. The quantification obtained via a principal components analysis shows patterns that could be targeted from the perspective of each care stakeholder so that systemic change can be induced. The matrix' heat mapping (Table 1) shows the importance of 'relationship', emphasizing in pink colour good practice, and identifying improvement opportunities in red colour (as explained in [12]). These care structures and depictions can deliver insights to individuals and institutions (micro-levels), they can be merged to reflect groups experiences (meso-levels) and be used to capture extended networks of end-users and therefore predict, model, and alter positive and negative patterns of FoC (macro-levels) [e.g.,12,14,15,16]

This approach can convey, track, and measure complex and emotive issues in a quantifiable manner. Further development of this method can aid the creation of multilevel solutions that align goals across all individuals, networks, and levels of complex health and care problems. Our team is envisioning user-friendly FoC matrixes embedded within digital technologies, using artificial intelligence to facilitate systemic solutions to improve health systems [e.g.,12,13,14]. The digitalised versions of the matrix are relevant for those who receive, provide, manage, or accredit care. This digitisation will support the many varied uses of the matrix, for example as a selfevaluation tool for caring teams and health practitioners, a quality and compliance tool, a patient and family feedback tool, a teaching and training tool, a caring journey tracker (of individual and institutions performance), a longitudinal data collection and reporting 
Table 1. Ms Merle Mitchel's statement analysed via the FoC Matrix

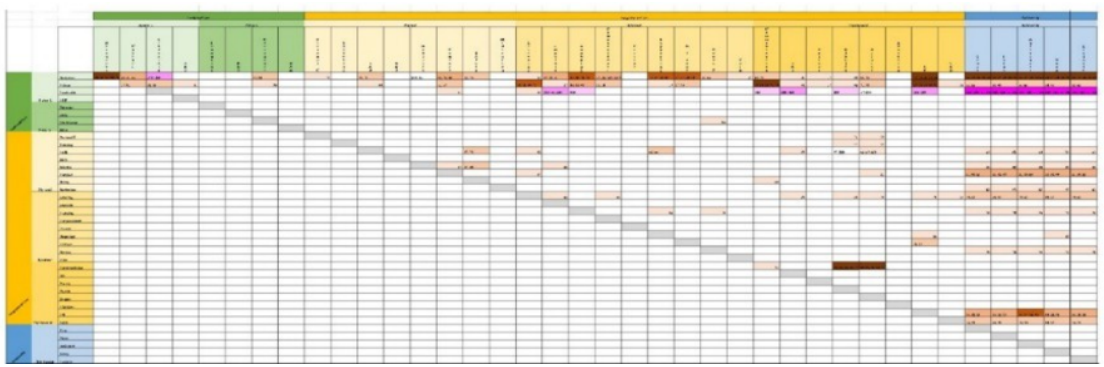

tool with Bayesian predictive functions, as per our connected endeavours $[12,14,15,16]$. These possibilities are investigated via a combination of projects conceptualised to codevelop healthcare, self-care, and caring solutions [12,13,14,15,16,17]. Our research program aims to support better lives, better communities, better care, and better systems.

\section{References}

[1] Clancy TR, Effken JA, Pesut D. Applications of complex systems theory in nursing education, research, and practice. Nursing outlook. (2008). Sep 1;56(5):248-56.

[2] Andersson, N, Silver H. (2019). Fuzzy cognitive mapping: An old tool with new uses in nursing research. Journal of advanced nursing.

[3] Kitson A, Brook A, Harvey G et al. Using complexity and network concepts to inform healthcare knowledge translation. International journal of health policy and management, (2018), 7(3), 231.

[4] Boissevain J. Network analysis: A reappraisal. Current anthropology 20.2 (1979): 392-394.

[5] Royal Commission into Aged Care Quality and Safety. Interim Report: Neglect. Commonwealth of Australia (2019). Last visited the 2/12/19.

[6] Wells Y, Brooke E, Solly KN. Quality and Safety in aged care virtual issue: What Australian research published in the Australasian Journal on Ageing tells us. Australasian journal on ageing, (2019), 38(1), E1-E6.

[7] Feo R, Kitson A, Conroy T. How fundamental aspects of nursing care are defined in the literature: A scoping review. Journal of clinical nursing 27.11-12 (2018): 2189-2229.

[8] Ackoff RL, Gharajedaghi J. Reflections on systems and their models. Systems Research, (1996),13(1), 13-23.

[9] Young RA, Roberts RG, Holden RJ. The challenges of measuring, improving, and reporting quality in primary care. The Annals of Family Medicine. (2017) Mar 1;15(2):175-82.

[10] Kitson A, Conroy T, Kuluski K, Locock L, Lyons R. Reclaiming and redefining the fundamentals of care: Nursing's response to meeting patients' basic human needs. (2013).

[11] Gross JL, Yellen J. Graph theory and its applications. CRC Press, (2005), Sep 22.

[12] Conroy T, Pinero de Plaza MA, Mudd A, Kitson A. May 2021, (Accepted/In press). Measuring fundamental care using complexity science principles: A descriptive case study of a methodological innovation. Journal of Clinical Nursing.

[13] Pinero de Plaza MA, Beleigoli A, Mudd A et al. (2021). Not well enough to attend appointments: Telehealth versus health marginalisation. In Studies in Health Technology and Informatics: Healthier Lives, Digitally Enabled (pp. 72-79). IOS PRESS.

[14] Pinero de Plaza MA, McMillan P, Buchanan R et al. (2021, March). Exclusion from primary healthcare: a barrier to the national disability strategy implementation. In Consumers Health Forum Virtual Summit 2021: Shifting Gears.

[15] Pinero de Plaza MA, Taghian M, Marmolejo-Ramos F, Barrera-Causil CJ, Hall J. (2021). Investigating salience strategies to counteract obesity. Health Promotion International.

[16] Pinero de Plaza MA. (2020): R code and related $\mathrm{R}$ files. figshare. Software. https://doi.org/10.6084/m9.figshare.13114601.v1

[17] Pinero de Plaza MA, Archibald M, Lawless M et al. (2021). PROLIFERATE: An adaptable framework to evaluate participatory research products. 